\title{
Nitrogen Phase Separation During Free Fall to Inform Design of Future Microgravity Cryogenic Rankine Power Cycles
}

\author{
Roberto J. Fernandez, Matthew J. Traum ${ }^{1}$ \\ Mechanical Engineering Department, Milwaukee School Of Engineering, Milwaukee, WI
}

\section{Introduction}

On Earth, potential solutions for our sustainable, enduring, high-density power needs are plentiful. However, in space no practical solutions currently exist to support long-term manned interplanetary missions. These missions require power sources possessing three critical attributes: high power density, longevity, and continuous operation independent of direct sunlight. Many existing space power technologies have two, but not all three of these critical elements.

The two Voyager spacecraft use radioisotope thermoelectric generators (RTGs), which are extremely robust and long-lived with 50-year operational lives. Of the three essential attributes for interplanetary manned space missions, RTG's possess two: longevity and operation independent of sunlight. However, their energy density is low. Newly produced RTG's generate about 120 watts of electrical power and weigh about $45 \mathrm{~kg}$, yielding an energy density of about $2.67 \mathrm{~W} / \mathrm{kg}$. ${ }^{\mathrm{i}}$ This low power density is far too small to support manned space missions.

Advanced fuel cells were used to power NASA's Orbiter. Of the three essential attributes for interplanetary manned space missions, fuel cells possess high energy density and can operate independent of sunlight. However, they do not possess longevity because their consumables are used up rapidly. The Orbiter's fuel cells were small in physical size and mass, able to produce 12 peak kilowatts, and they boasted energy density exceeding $529 \mathrm{~W} / \mathrm{kg}$. ${ }^{\text {ii }}$ Nonetheless, these cells required routine maintenance and used consumable supplies of oxygen and hydrogen. Orbiters typically contained 3 fuel cells each with an operating life of 2000 hours. Each fuel cell consumed on average $1.8 \mathrm{~kg}$ of oxygen and $0.27 \mathrm{~kg}$ of hydrogen per hour. ${ }^{\text {iii }}$ About $11,000 \mathrm{~kg}$ of oxygen and $1600 \mathrm{~kg}$ of hydrogen needed to be carried by the Orbiter for full operation of all three fuel cells. Use of fuel cells over the prolonged periods required for manned interplanetary missions would require massive fuel storage systems which are not feasible.

The Photovoltaic Cells (PVCs) making up the solar arrays of the International Space Station are the current answer to sustained power generation for manned space missions. Of the three essential attributes for interplanetary manned space missions, PVCs possess relatively high energy density as well as longevity. However, they require sunlight to operate. Sunlight intensity falls off as the square of the distance from the source. Thus, PVCs will produce significantly less power as interplanetary spacecraft travel away from the sun. For example, the semi-major radius of Earth's orbit versus Mar's orbit is

\footnotetext{
${ }^{1}$ The corresponding author gratefully acknowledges the Wisconsin Space Grant Consortium 2012-2013 Higher Education Incentives Award Program for making this project possible. Funding was also provided by the Texas Space Grant Consortium 2010-2011 New Investigator Award Program. Both authors acknowledge Cameron R. Schulz for his helpful consultation on the payload free fall model. This paper's undergraduate lead author is a member of the Milwaukee Undergraduate Researcher Incubator (MURI) at MSOE, an organization which fast-tracks undergraduates into meaningful early research experiences.
} 
about 74,800,000 km versus $114,000,000 \mathrm{~km}$ respectively, meaning a PVC on a spacecraft orbiting Mars could produce only about $43 \%$ of the power it could while orbiting Earth. The other shortcoming of PVCs is need for large collector surface area. Due to their size, large arrays are expensive to launch into space. Hence power per unit area is the chief comparison metric to evaluate these systems. The solar array network on the International Space Station is 4047 square meters and produces 90 peak kilowatts of power, ${ }^{\mathrm{iv}}$ which equates to about $22.2 \mathrm{~W} / \mathrm{m}^{2}$.

Realizing that all conventional space power generation technologies lack at least one essential attribute for manned interplanetary missions, NASA identified Rankine cycle power plants as an essential element for future manned space missions. If operated from a nuclear heat source, these power cycles could provide high energy density, longevity, and continuous operation independent of direct sunlight. ${ }^{\mathrm{V}}$ In fact, recent advances in micro-fabrication technology suggest Rankine cycles could be produced with predicted power densities of $12,000 \mathrm{~W} / \mathrm{kg}$. ${ }^{\text {vi }}$ The Rankine cycle is the same thermodynamic power cycle used in conventional terrestrial coal-fired power plants for electricity generation.

\section{Background}

One promising avenue for space-based power generation for manned interplanetary missions is the cryogenic Rankine power cycle. This power cycle is used on Earth by utilities within the Liquid Natural Gas (LNG) industry to recover energy otherwise lost via LNG vaporization to supply natural gas to end users. ${ }^{\text {vii }}$ Given NASA's vast experience handling cryogenic fluids in space, nearly all the technologies to implement a space-based Rankine power cycle already exist. ${ }^{\text {viii }}$ The largest element by area of a spacebased Rankine power cycle would be the condenser, which could be integrated into the outer surface of a spacecraft to radiate entropy to space. This configuration, if fired by a nuclear reactor, would boast a much larger power to unit area ratio than PVCs, and would represent a viable solution to the need for high-density power generation in space for manned interplanetary missions. ${ }^{\text {ix }}$

\section{Theory}

The hallmark of the Rankine cycle is two-phase (liquid/vapor) working fluid. As it flows through the cycle, the working fluid is vaporized in the evaporator, expanded through the turbine, condensed in the condenser, and then pumped back into the evaporator. Critical to implementing a space-based Rankine cycle is handling microgravity phase separation in the condenser. Working fluid that has liquefied can be pumped back to the evaporator, but any remaining vapor must be separated and re-condensed before it can be pumped. On Earth, Rankine cycle liquid/vapor separation occurs naturally via gravity-induced buoyancy; with gravity absent in space, this separation mechanism will not work. Thus, to implement any space-based Rankine power cycle, a need exists to perfect microgravity phase separation.

Cryogenic fluid microgravity phase separation research with porous barriers has been conducted. ${ }^{\mathrm{x}}$ Both NASA and the U.S. military have perfected cryogenic fluid management techniques, including phase separation, for space flight functions including life support, fuel management, and supply transfer. ${ }^{\text {xi } x i i}$ Previous microgravity cryogenic fluid phase separators utilized thermo-mechanical effect, a super-fluid property of helium II, to phase separate fluid across a porous plug. ${ }^{\text {xiii,xiv,xv }}$ Certain porous plugs were also found to be efficient microgravity phase separators for helium I, which is not a superfluid. ${ }^{\text {xvi }}$ Thus, phase separation of other cryogenic fluids, such as liquid nitrogen, can be achieved by applying findings demonstrated for helium I. 
A porous plug will be used as the phase separator within the cryogenic Rankine cycle condenser. The porous plug will have capillaries on the order of a few micrometers in diameter inlayed within it that serve as passages for the fluid. The plug shall also have a thickness equal to $4 \%$ of the diameter of the condenser. The thickness of the plug is an important variable because as the fluid travels through the barrier it will encounter viscous and capillary forces that retard motion, and it is desirable to minimize these energy loss mechanisms. The thicker the plug, the larger these retarding forces become making the pumping process more difficult and therefore less efficient. ${ }^{\text {viii }}$ A diagram of the force interactions across the barrier is given in Figure 1.

While cryogenic fluid phase separation using a porous plug is a proven concept, practical and efficient implementation of this idea within a spacebased power plant is dependent upon optimizing many underlying variables: nitrogen thermal-fluidic properties such as contact angle, density, surface tension, and viscosity; as well as porous plug properties like material selection, void fraction, capillary size, interior surface roughness, and tortuosity. While some of these properties can be measured directly, many cannot. Moreover the effects of interactions between properties are complex and poorly understood. Therefore, we opt to proceed toward choosing the best porous barrier for cryogenic fluid microgravity phase separation via an empirical Design Of Experiments (DOE) approach.

\section{Proposed Experiment}

The experiment will rely upon a method of simulating microgravity shown in Figure 2 . The phase separation efficacy of different porous plugs will be tested by launching a sounding rocket one mile (about 1609 meters) high. At apogee, the rocket will separate into two pieces: the rocket shell itself and a smaller payload pod containing the experimental apparatus. Experimental measurements will be taken in the payload pod, which will be designed to minimize drag, throughout its descent. While an object is accelerating during free fall, its contents are subjected to microgravity; the instant the falling object reaches terminal velocity, gravitational forces are balanced by drag forces and microgravity conditions cease in its interior. Therefore, the experiment will end once the rocket has attained terminal velocity.

To approximate how long the free-fall period will last, the kinematic equation for one-dimensional motion of an object falling under gravity is to be utilized. These equations are applied assuming gravitational acceleration, $\mathrm{g}$, is independent of altitude; velocity of the pod at rocket apogee is zero; and that there is no drag on the payload pod: 


$$
\begin{gathered}
y(t)=y_{o}-\frac{1}{2} g t^{2} \\
v(t)=-g t
\end{gathered}
$$

where $y(t)$ and $v(t)$ are the pod altitude and velocity as a function of time, $\mathrm{t}$, and $\mathrm{y}_{\mathrm{o}}$ is the rocket apogee altitude. The assumptions used, particularly ignoring drag, oversimplify the model, but they still provide a reasonable order-of-magnitude estimate of the time available to complete experimental measurements. Critically, this time is on the order of seconds, which is a reasonable duration during which thermodynamic measurements such as temperature and pressure can be taken.

The payload pod will be designed so its parachutes deploy 150 meters above the ground, allowing it to slow to a safe impact velocity for successful recovery. Under the stated modeling assumptions, the payload pod will fall for about 17.25 seconds traveling over 1459 meters and reaching a velocity of over $169 \mathrm{~m} / \mathrm{s}$ at the time of chute deployment.

The total number of possible flights will be limited by cost, time, and the repeated successful launch and recovery of both the rocket and its payload. Therefore an empirical Design Of Experiments (DOE) approach will be used to minimize the number of flights needed to obtain information about how various porous plug parameters impact microgravity phase separation. For each test, the thermodynamic state of the nitrogen will be fixed by ensuring two independent intensive thermodynamic variables (temperature and specific volume) are identically reproduced each time. Porous plugs will be selected that exhibit four levels each of three different important independent variables: material selection, void fraction, and internal capillary size. To run every

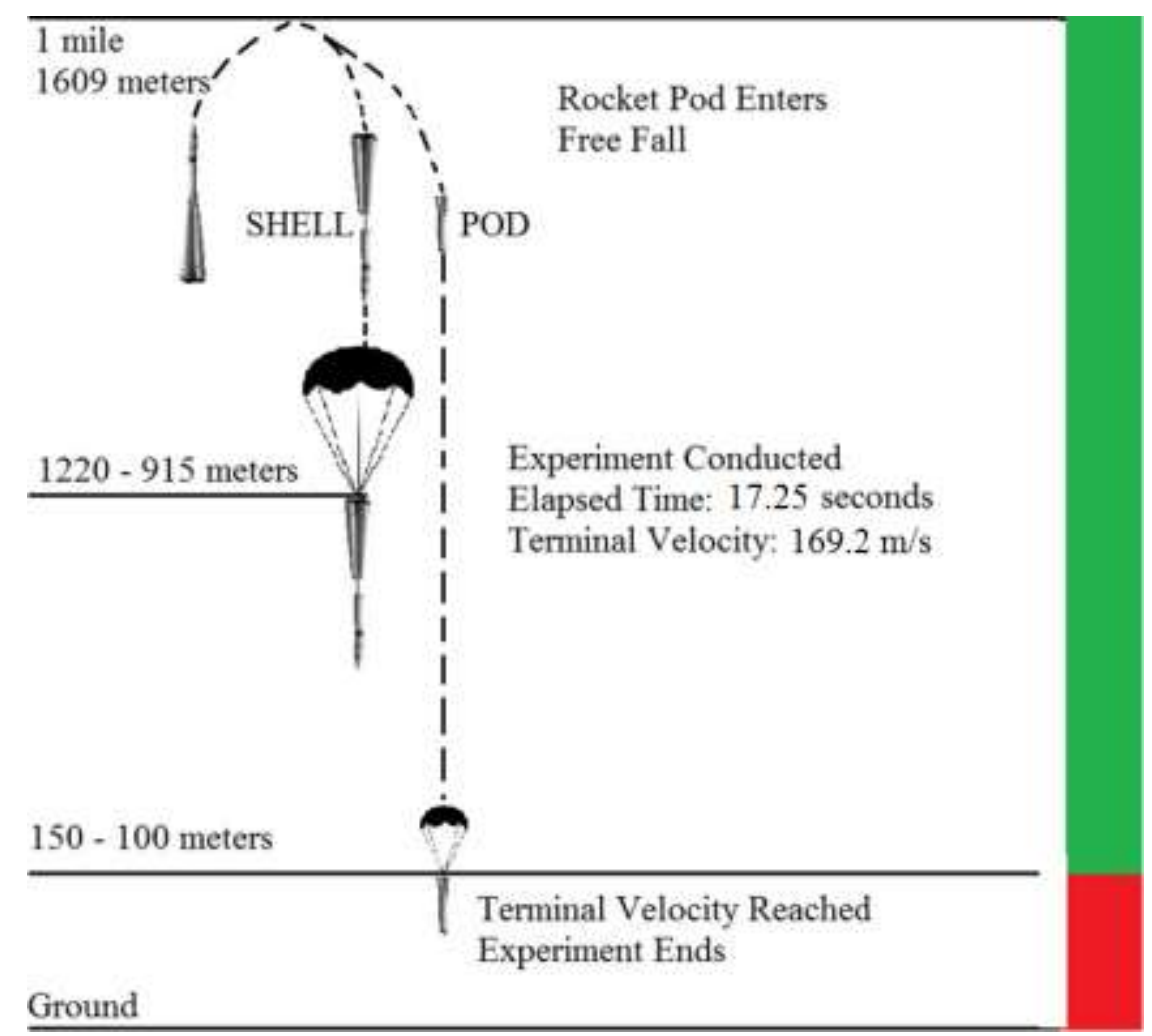

Figure 2: Rocket launch and recovery cycle for the proposed microgravity porous plug cryogenic fluid phase separation experiment.

test in this experiment matrix would require $4^{3}=64$ unique flights. However, using an orthogonal array following Taguchi's method for experiment reduction, only 16 launches will be needed to fully explore the experimental space. The results from these tests will be used to select the best combination of porous plug parameters for use in microgravity cryogenic Rankine power cycles to enable nitrogen liquid/vapor phase separation in the condenser. 


\section{Conclusions}

The state-of-the-art with respect to conventional space-based power generation technologies was outlined in this paper. Since none of the current technologies poses all three attributes critical for future manned interplanetary missions; high power density, longevity, and continuous operation independent of direct sunlight; none are viable for these missions. Some new type of space-based power generation technology is needed to enable future planned manned missions beyond Earth orbit. The cryogenic Rankine cycle is a strong candidate since all technologies and techniques needed to implement this power cycle have already been developed and demonstrated. To function correctly, Rankine cycles require liquid/vapor phase separation, a process normally accomplished in terrestrial applications via gravity-driven buoyancy. For a space-based Rankine cycle to be successfully implemented, microgravity phase separation is needed.

NASA and the U.S. military have already demonstrated microgravity phase separation of non-superfluid cryogenic liquid-vapor mixtures using porous plugs. This technique will therefore be adapted to implement phase separation for a cryogenic Rankine cycle. To select the best combination of porous plug parameters to minimize pressure losses (and parasitic power losses) in the cycle a series of experiments will be conducted to test the phase separation efficacy of several porous plug varieties. An experimental apparatus to measure phase separation efficacy will be launched on a sounding rocket and allowed to free fall, producing microgravity conditions for 17.25 seconds during which the phase separation efficacy of each porous plug sample will be tested and evaluated.

Using the DOE technique following Taguchi's method for experiment reduction, the best performing combination of porous barrier parameters (material selection, void fraction, and internal capillary size) will be identified that demonstrates the most effective nitrogen liquid/vapor phase separation in microgravity. This experiment will provide the final technology demonstration needed to fully implement a cryogenic Rankine power cycle for space-based power generation applications.

\section{References}

\footnotetext{
i NASA, "Space Radioisotope Power Systems Multi-Mission Radioisotope Thermoelectric Generator," URL: http://solarsystem.nasa.gov/docs/MMRTG_Jan2008.pdf, accessed 8/15/2013.

ii R. E. Martin, "Lightweight Fuel Cell Powerplant Components Program," Report NASA-CR-161412, United Technologies Power Systems Division, February, 1980.

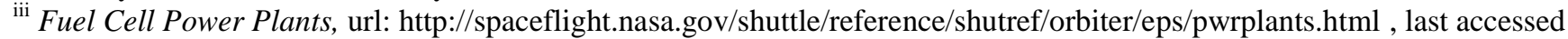
$8 / 15 / 2013$.

${ }^{\text {iv }}$ Facts and Figures, url: http://www.nasa.gov/mission_pages/station/main/onthestation/facts_and_figures.html\#.Uf8A2JLVB8E, last accessed 8/15/2013.

${ }^{v}$ R. T. Lahey, V. Dhir, "Research in Support of the Use of Rankine Cycle Energy Conversion Systems for Space Power and Propulsion," Report NASA/CR-2004-213142, 2004.

${ }^{v i}$ L. G. Fréchette, C. Lee, S. Arslan, Y.-C. Liu, "Design of a Microfabricated Rankine Cycle Steam Turbine for Power Generation,” Paper IMECE2003-42082, Proceedings of IMECE '03 the 2003 ASME International Mechanical Engineering Congress \& Exposition, Washington, D.C., November 16-21, 2003.

${ }^{\text {vii } C r y o g e n i c}$ Power Generation System Recovering LNG's Cryogenic Energy and Generating Power for Energy and CO Emission Savings, url: http://www.osakagas.co.jp/en/rd/technical/1198907_6995.html, last accessed 8/15/2013.

viii J. E. McKeathen, R. F. Reidy, S. K. S. Boetcher, M. J. Traum, "A Cryogenic Rankine Cycle for Space Power Generation," AIAA Paper Number 2009-4247, Proceedings of the 41st AIAA Thermophysics Conference, San Antonio, TX, June 22 - 25, 2009.
} 
${ }^{i x}$ L. Lin, X.-L. Yu, Z. Xin, X.-Z. Zhang, “Thermal Cycle Design of Liquid Nitrogen Engine,” Journal of Zhejiang University, Vol. 40, No. 11, 2006, pp. 1989-1993.

${ }^{x}$ D. M. Althausen, F. D. Quinn Jr., C. T. Bunnell, N. E. Daidzic, "Porous Media Condensing Heat Exchanger with Integral Gas Liquid Separation for Space Flight Use," Proceedings of the 44th AIAA Aerospace Sciences Meeting, American Institute of Aeronautics and Astronautics Inc., Reston, VA, 2006, pp. 16186-16196.

${ }^{x i}$ M. J. DiPirro, R. F. Boyle, O. Figueroa, D. Lindauer, D. C. McHugh, P. J. Shirron, "Superfluid Helium Heat Transfer," AIAA/ASME Thermophysics and Heat Transfer Conference. Published by ASME, New York, 1990.

xii M. J. DiPirro, J. Zahniser, "Liquid/Vapor Phase Boundary in a Porous Plug," Proceedings of the 1989 Cryogenic Engineering Conference, Vol. 35, Part 1, 1990, pp. 173-180.

${ }_{\text {xiii }}$ M. J. DiPirro, R. F. Boyle, O. Figueroa, D. Lindauer, D. C. McHugh, P. J. Shirron, "The SHOOT Cryogenic System," Superfluid Helium Heat Transfer, United Engineering Center, New York, NY, 1990, p. 29.

${ }^{\text {xiv }}$ M. J. DiPirro, J. Zahniser, "The Liquid/Vapor Phase Boundary in a Porous Plug," Advances in Cryogenic Engineering, Vol. 35, Plumum Press, New York, NY, 1990, pp. 173-180.

${ }^{x v}$ V. G. Baranov, A. I. Vinnikov, M. I. Gutkin, "A Study of the Operation of a Helium Vessel Phase Separator," Translated from Khimicheskoe I Neftaynoe Mashinostroenie, No. 8, August 1976, pp. 12-13.

${ }^{x v i}$ M. J. DiPirro, D. C. McHugh, J. Zahniser, "Phase Separators for Normal and Superfluid Helium," Proceedings of the Twelfth International Cryogenic Engineering Conference - ICEC 12, 1988, pp. 681-689. 